\title{
Shortcuts to adiabaticity in cutting a spin chain
}

\author{
Feng-Hua Ren ${ }^{1,2}$, Zhao-Ming Wang $^{1}{ }^{*}$ and Yong-Jian $\mathrm{Gu}^{1 \dagger}$ \\ ${ }^{1}$ Department of Physics, Ocean University of China, Qingdao 266100, China and \\ ${ }^{2}$ School of Computer Engineering, Qingdao Technological University, Qingdao 266033, China
}

(Dated: October 17, 2016)

\begin{abstract}
"Shortcuts to adiabaticity" represents a strategy for accelerating a quantum adiabatic process, is useful for preparing or manipulating a quantum state. In this paper, we investigate the adiabaticity in the dynamics of an $X Y$ spin chain. During the process of cutting one long chain into two short chains, a "shortcut" can be obtained by applying a sequence of external pulses. The fidelity which measures the adiabaticity can be dramatically enhanced by increasing the pulse strength or pulse duration time. This reliability can be kept for different types of pulses, such as random pulse time interval or random strength. The free choice of the pulse can be explained by the adiabatic representation of the Hamiltonian, and it shows that the control effects are determined by the integral of the control function in the time domain.
\end{abstract}

PACS numbers: 03.65.-w,42.50.Dv,75.10Jm

\section{INTRODUCTION}

The quantum adiabatic principle is a fundamental concept in quantum mechanics $[1,2]$. This principle states that for an infinitely slow (adiabatic) transformation the eigenstate population of a time-dependent Hamiltonian $H(t)$ is constant in time. The quantum adiabatic principle proves very useful in manipulating states and shows a wide variety of applications in quantum information processing, such as quantum algorithm design and implementation [3], universal adiabatic and holonomic quantum computation $[4,5]$, and quantum state transfer $[6-$ $12]$.

However, the adiabatic process is necessarily slow, since it requires an infinitely long evolution time $T$, and hence can suffer from dissipation and noise. Therefore "Shortcuts to adiabaticity" is suggested and it represents a finite time process which yields the same final state as an adiabatic, infinitely slow process. It has recently attracted more and more attention. In particular, in quantum computing $[13,14]$ and specifically, in quantum annealers [15-17] these shortcuts prove to be extremely useful. Among the known methods for shortcuts, the transitionless driving $[18,19]$ or the counteradiabatic control [20] are based on constructing modification of an original Hamiltonian to compensate for nonadiabatic errors. The Rapid Adiabatic Passage [21-23] (RAP) or the Stimulated Ranman adiabatic passage [24-29] (STIRAP) techniques can also be used to manipulate the state of a quantum system with interacting fields, e.g. coherently population transfer between the two ground states of a three energy level atoms [24]. Recently, STIRAP in a three-level superconducting circuit [30] is experimentally studied. By using two adiabatic Gaussian-shaped control microwave pulses, a population transfer efficiency

\footnotetext{
*mingmoon78@126.com

†yjgu@ouc.edu.cn
}

$>80 \%$ between the ground state and the second excited state is obtained. In the strong field regime, Adiabatic Passage by Light-Induced Potentials (ALIP) was introduced [31, 32]. By using ALIP, a wave packet can be transferred from one molecular potential to the displaced ground vibrational state of another [33]. Another prominent method is to apply a sequence of pulses upon the system [13, 14, 34, 35] during the evolution, consequently the adiabatic passage can be sped up. This method has some similarities with the above mentioned RAP or STIRAP techniques, such as they both use time-dependent field, relies on the instantaneous eigenstate of the system's Hamiltonian, etc.

In this paper, we propose here a shortcut to adiabatic passage by using the pulse control technique $[13,14,34]$. As an example, we study the dynamical process of cutting a long spin chain into two short chains. Suppose initially the long chain stays at one of its eigenvectors, we find that by introducing pulse control, the evolution of the initial state will remain close to the desired adiabatic eigenvector during the cutting process even in a non-adiabatic regime. The pulses can be represented as a control function. Significantly, the integral of the control function in the time domain determines the effectiveness of the control. Thus, the particular design of the control function is not necessary. Both regular and random pulse sequences can be used to speed up the adiabatic passage.

\section{MODEL AND METHOD}

We now consider a one-dimensional (1D) $X Y$ model $[43,44]$

$$
\begin{aligned}
H_{0}(t)= & \sum_{i=1, i \neq j}^{N-1} J_{i, i+1}\left(X_{i} X_{i+1}+Y_{i} Y_{i+1}\right) \\
& +J_{j, j+1}(t)\left(X_{j} X_{j+1}+Y_{j} Y_{j+1}\right),
\end{aligned}
$$

where $J_{i, i+1}$ is the coupling strength between sites $i$ and $i+1$. Now suppose the couplings between sites $j$ and 


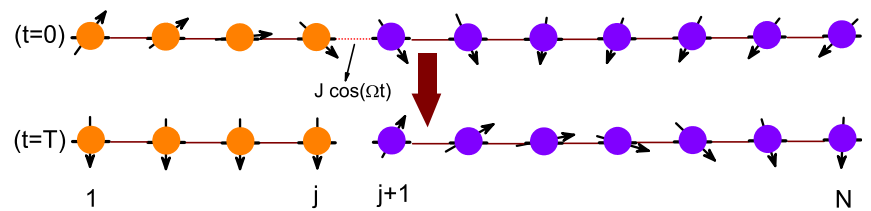

FIG. 1: (Color online) Sketch of the perfect adiabatic evolution. The one-dimensional spin chain has uniform coupling strength $J$ except that $J_{j, j+1}=J \cos \Omega t$ between sites $j$ and $j+1$. The number of excitations (magnons) conserves during the evolution. At $t=0$, the chain has been cooled to its ground state which is in the "one-magon" state. For an infinitely long time $t=T$, the chain has been cut into two chains: one short chain with $j$ spins and one long chain with $N-j$ spins. Now the magon is totally in the long chain and the spins in the short chain are all at spin down states. Experimentally this setup can be realized by $1 \mathrm{D}$ optical lattice created by laser beams (see [41, 42]).

$j+1$ are cut little by little, and finally it becomes zero (see Fig. 1). $X_{i(j)}, Y_{i(j)}$ are the Pauli matrices acting on spin $i(j)$.

We set

$$
J_{i, i+1}=\left\{\begin{array}{c}
J,(i \neq j) \\
J \cos (\Omega t),(i=j)
\end{array}\right.
$$

where $\Omega=\pi /(2 T)$ and $T$ is the total evolution time. We will use a ferromagnetic coupling, and take $\hbar=1, J=-1$ throughout. From $t=0$ to $t=T, J_{j, j+1}$ becomes from -1 to zero. At the same time one long chain with $N$ spins has been cut to two short chains with $j$ spins and $N-j$ spins, respectively. Due to $\left[\sum_{i} Z_{i}, H_{0}(t)\right]=0$, the $\mathrm{z}-$ component of the total spins or the magnon is conserved during the time evolution. For simplicity, we only discuss the problem in the "one-magnon" subspace where the total number of up spins is one. Experimentally this model could be realized by the ultracold bosons in $1 \mathrm{D}$ optical lattice, as in the hard-core limit it can be mapped into an effective spin 1/2 XY model [36]. An optical lattice can be created by using a retroreflected laser beam and the bosons are also subjected to a transverse confinement for realizing the $1 \mathrm{D}$ configuration (see [37, 38] for details). The couplings $J$ could be tuned individually by focusing additional laser beams perpendicular to the lattice direction for different sites. It is easy to create uniform couplings by using this technique while for creating arbitrary couplings for ultracold atoms, it might be feasible to use the spatial light modulator technology [39, 40]. Thus the coupling $J_{j, j+1}$ could be tuned experimentally.

The instantaneous eigenvectors and eigenvalues of $H_{0}(t)$ can be denoted as

$$
H_{0}(t)\left|\psi_{m}(t)\right\rangle=E_{m}(t)\left|\psi_{m}(t)\right\rangle \text {. }
$$

They constitute (at any particular instant) an orthonormal set $\left\langle\psi_{n}(t) \mid \psi_{m}(t)\right\rangle=\delta_{n m}$ and they are complete. (a)

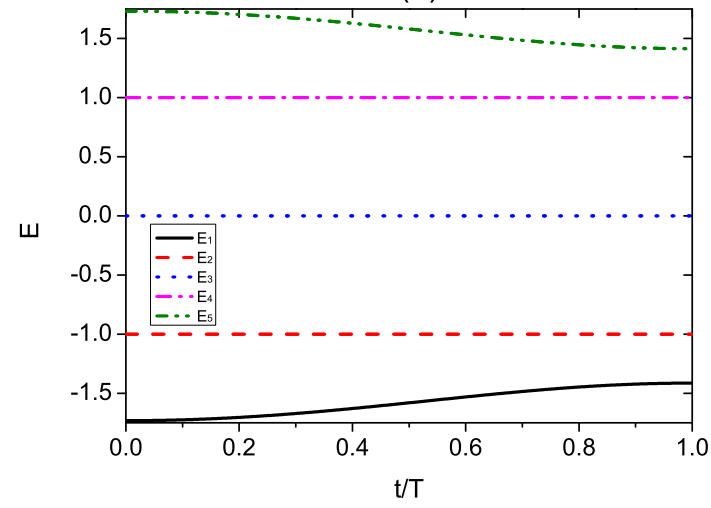

(b)

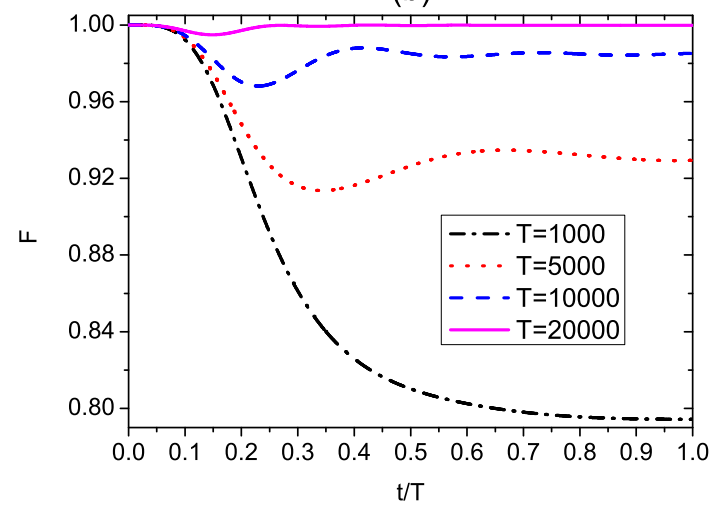

FIG. 2: (Color online) (a) The eigenenergies $E_{n}(t)$ of the eigenvectors for the instantaneous Hamiltonian $H_{0}(t)$ as a function of $t / T, N=5, J_{2,3}=J \cos (\Omega t) . E_{1}(t)$ (solid line) is the ground state energy and $E_{5}(t)$ (dash-dot-dot line) is the highest energy. (b) The fidelity $F=\left|\left\langle\Psi(t / T) \mid \psi_{1}(t / T)\right\rangle\right|$ as a function of $t / T$ for different evolution time $T$. $|\Psi(t / T)\rangle$ is the wave function governed by the time-dependent Schrödinger equation and $\left|\psi_{1}(t / T)\right\rangle$ is the instantaneous ground state of the time-dependent Hamiltonian $H(t) . \quad N=125, J_{50,51}=$ $J \cos \Omega t$, the time interval (step length) that we used to calculate is taken as $\tau=0.5$.

We first consider the perfect adiabatic evolution, which corresponds with an infinitely long evolution time $T$. At $t=0(t=T)$ the Hamiltonian $H_{0}(0)\left(H_{0}(T)\right)$ has analytic expression of eigenvalues $E_{m}=2 J \cos q_{m}$ with the corresponding eigenvectors [45]

$$
\left|\psi_{m}\right\rangle=\sqrt{2 /(N+1)} \sum_{j=1}^{N} \sin \left(q_{m} l\right)|\mathbf{l}\rangle,
$$

where $q_{m}=\pi m /(N+1), m=1,2, . . N . \quad|\mathbf{l}\rangle$ denotes that the chain is at a state where the site $l$ is at spin up state $|1\rangle$ and all other sites are at spin down state $|0\rangle$. Suppose initially the system is at the ground state $\left|\psi_{1}(0)\right\rangle$. According to the adiabatic theorem, the system will go along the passage of the instantaneous ground state. Then the general solution to the time-dependent Schrödinger equation $i|\Psi(t)\rangle=H(t)|\Psi(t)\rangle$ can be expressed as $|\Psi(t)\rangle=\left|\psi_{0}(t)\right\rangle$, here we have neglected the 
difference of the phase factor between the the two states.

From Eq. (2), for infinitely long time $T, J_{j, j+1}=0$, the long chain with $N$ spins has been cut into two parts, one has $j$ spins with energy $E_{j, m}=2 J \cos [\pi m /(j+1)]$ and the other has $N-j$ spins with energy $E_{N-j, m}=$ $2 J \cos [\pi m /(N-j+1)]$. Clearly if $j<(N-j), m=1$ (ground state), $E_{j, 1}<E_{N-j, 1}$, the magnon will be in the long chain. All the spins in the short chain will be at spin down states (zero-magnon state). In the whole paper, we will always assume $j<(N-j)$, i.e., one short chain with $j$ spins and one long chain with $N-j$ spins.

For example, $N=5$, at $t=0$, the initial state is

$$
\left|\psi_{1}(0)\right\rangle=\sqrt{\frac{1}{3}}\left(\frac{1}{2}|\mathbf{1}\rangle+\frac{\sqrt{3}}{2}|\mathbf{2}\rangle+|\mathbf{3}\rangle+\frac{\sqrt{3}}{2}|\mathbf{4}\rangle+\frac{1}{2}|\mathbf{5}\rangle\right) .
$$

Now we cut the coupling between sites 2 and 3 . At $t=T$, from Eq. (4) the final state is

$$
|\Psi(T)\rangle=\left|\psi_{1}(T)\right\rangle=\frac{1}{2}|\mathbf{3}\rangle+\frac{\sqrt{2}}{2}|\mathbf{4}\rangle+\frac{1}{2}|\mathbf{5}\rangle .
$$

In this case, the state in the short chain will be at $|00\rangle$, now the magnon has been kept in the long chain. Fig. 2(a) plots the energy spectrum as a function of parameter $t / T$. The ground state energy changes from $E_{1}(0)=-\sqrt{3}$ to $E_{1}(T)=-\sqrt{2}$ as expected. Note that the energies $E_{2}(t), E_{3}(t)$ and $E_{4}(t)$ do not change with time.

For finite $T$, the system will not go along an adiabatic passage, the adiabaticity is destroyed. We use the fidelity $F=\left|\left\langle\Psi(t) \mid \psi_{m}(t)\right\rangle\right|$ to measure the adiabaticity, where $\left|\psi_{m}(t)\right\rangle$ is the instantaneous eigenstate of the time-dependent Hamiltonian and $|\Psi(t)\rangle$ is the wave function governed by the the time-dependent Schrödinger equation.

The time evolution of the system proves hard to calculate due to the time-dependent Hamiltonian $H_{0}(t)$. Now we present numerical solution by using Trotter Approximation [46], the time-ordering unitary evolution operator driven by the time-dependent Hamiltonians $H_{0}(t)$ can be implemented as

$$
U(k \tau) \approx e^{-i H_{0}(k \tau) \tau} \ldots e^{-i H_{0}(2 \tau) \tau} e^{-i H_{0}(\tau) \tau},
$$

up to order $O\left(\tau^{2}\right) . \tau$ is the time interval (step length) that we used to calculate. $k \tau$ is the total evolution time.

Fig. 2(b) plots the fidelity $F$ as a function of parameter $t / T$ for different evolution time. We take $N=125$, $J_{50,51}=\cos \Omega t$. The results show that $F$ decreases monotonically with time for a short $T$. When $T \geq 20000$, $F>0.999$, the system enters into the adiabatic regime.

\section{NEAR PERFECT ADIABATIC EVOLUTION UNDER CONTROL}

Although the adiabaticity can be obtained for a long $T$, normally for performing quantum information processing (a)

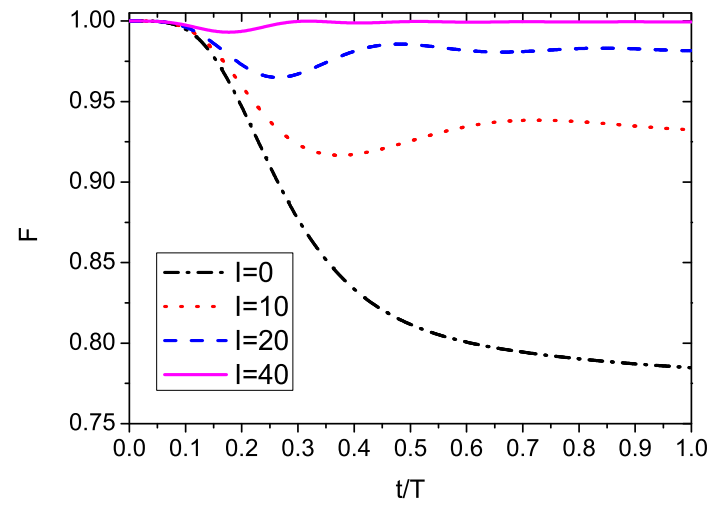

(b)

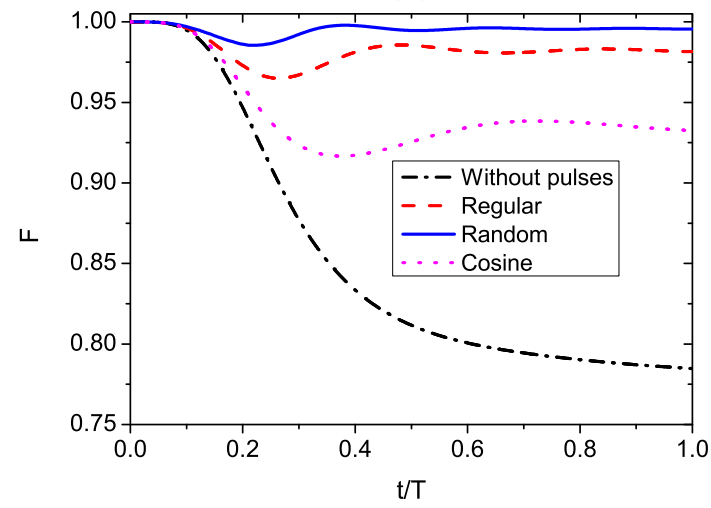

FIG. 3: (Color online) Dynamics of fidelity $F$ for (a) different strength ratio $I, m=n=5$; (b) four types of pulses: without pulses; regular rectangular pulses: $m=n=10$; random pulses with $I \in[10,30]$ and the ratio $n / m \in[0,1], m+n=$ 20; a fast cosine signal $c(t)=20 \cos ^{2}(20 t)$. For both cases, $N=100, J_{40,41}=J \cos \Omega t, T=500, \tau=0.5$. We apply the control during the time interval $m \tau$ and for time interval $n \tau$ it corresponds with free evolution.

tasks we need to use a short runtime. We next provide a protocol for "shortcuts to adiabaticity". Our strategy is to add an external control upon the Hamiltonian during the dynamical process. This is a generalization of that which was presented in Refs. [13, 34]. The dressed Hamiltonian is given by

$$
H(t)=[1+c(t)] H_{0}(t),
$$

where $c(t)=C(t) / J$. $C(t)$ is the above mentioned control function. It can be a sequence of fast signals. $c(t)$ is a dimensionless parameter and it measures the ratio of the control pulse strength to the coupling strength. In Ref [34], $C(t)$ could be a noise signal in a magnetic field. Ref [13] shows that this strategy can be used to accelerate quantum computation for EC3 problems. We will show that the adiabaticity can be enhanced for a proper choice of $C(t)$. If $C(t)$ is strong enough, the system will behave the same as in the adiabatic case. First we consider $C(t)$ is just a sequence of period rectangular pulses. Suppose $c(t)=I$ in the time interval $m \tau$ under control, 
$c(t)=0$ in the time interval $n \tau$ under free evolution. $m, n$ are integers. $(m+n) \tau$ is the width of the pulse in one period.

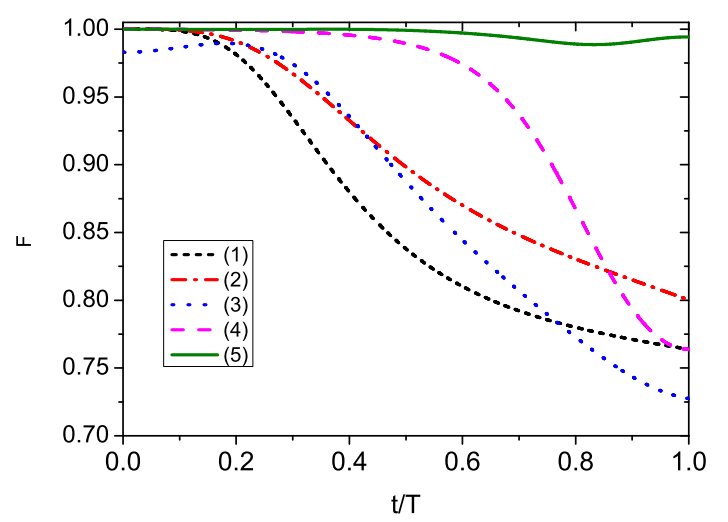

FIG. 4: (Color online) Dynamics of fidelity $F$ for different cutting or connecting methods. $N=50, T=50, m=n=$ $10, \tau=0.01$.

Now we consider the pulse strength effects on the acceleration of the adiabaticity. Fig. 3(a) shows that fixing the period $T=500$ in a non-adiabatic regime, the fidelity can be enhanced by gradually increasing $I$. We see that when $I>40$, i.e., the pulse strength which are used to speed up the breaking of the chain is about a factor of 40 larger than the coupling strength $J$, the adiabaticity can be induced and the fidelity is approaching one.

It might be not easy to create a sequence of pulses with equal period and strength. Next we will consider different types of pulses. In Fig. 3(b) we plot the dynamics of the fidelity for random and cosine signals $c(t)=20 \cos ^{2}(20 t)$, respectively. We also plot the cases without and with regular pulses as a reference. For random pulses, we calcu- late (thousands times) average density matrices and the corresponding average fidelity. Fig. 3(b) shows that the adiabaticity can be enhanced by applying the random or cosine pulses, especially the fidelity of the random pulses is higher than the regular.

We now consider what happens for the other cutting methods of the chain. In Fig. 4 for $N=50, T=$ 50 we plot the fidelity dynamics for five cases: (1) $J_{20,21}=J \cos \Omega t$ (cutting into two chains); (2) $J_{20,21}=$ $J_{45,46}=J \cos \Omega t$ (cutting into three chains); $(3) J_{20,21}=$ $J \cos \Omega t, J_{45,46}=J \sin \Omega t$ (cutting and connecting two chains); (4) $J_{20,21}=J \sin \Omega t$ (connecting two chains into one chain); (5) $J_{20,21}=J \sin \Omega t, I=50, n=m=10, \tau=$ 0.01 (connecting with pulses). We find that for case (1)(4), without pulses they all decrease gradually with time in a non-adiabatic regime $T=50$, especially we obtain a highest fidelity at $t=T$ when cutting the chain into three pieces [case (2)] compared with other cases (1), (3) and (4). We conjecture that the more pieces we cut, the higher fidelity we will obtain. The reason is that for a shorter chain it needs shorter time to get into the adiabatic regime. Note that for cases (1) and (4) which corresponds with cutting and connecting the coupling $J_{20,21}$, the two dynamics curves starts from the same value and ends with the same value. While in the middle regime, the fidelity in case (4) is always higher than in case (1), this can also be explained as shorter chain is easier to attain adiabaticity. Again the fidelity can be enhanced by increasing $I$. When connecting two chains [case (5)] under pulse control, the fidelity is approaching one for $I=50$.

To understand the above acceleration mechanism, we write the adiabatic representation of the Hamiltonian in Eq. (8). $H(t)$ in a adiabatic basis can be denoted as

$$
H(t)=-i\left(\begin{array}{cccc}
\left\langle\psi_{1} \mid \dot{\psi}_{1}\right\rangle & \left\langle\psi_{1} \mid \dot{\psi}_{2}\right\rangle e^{-i C_{21}(t)} & \ldots & \left\langle\psi_{1} \mid \dot{\psi_{N}}\right\rangle e^{-i C_{N 1}(t)} \\
\left\langle\psi_{2} \mid \dot{\psi}_{1}\right\rangle e^{i C_{21}(t)} & \left\langle\psi_{2} \mid \dot{\psi}_{2}\right\rangle & \ldots & \left\langle\psi_{2} \mid \dot{\psi}_{N}\right\rangle e^{-i C_{N 2}(t)} \\
\ldots & \ldots & \left\langle\psi_{m} \mid \dot{\psi_{n}}\right\rangle e^{-i C_{m n}(t)} & \ldots \\
\left\langle\psi_{N} \mid \dot{\psi}_{1}\right\rangle e^{i C_{N 1}(t)} & \left\langle\psi_{N} \mid \dot{\psi}_{2}\right\rangle e^{i C_{N 2}(t)} & \ldots & \left\langle\psi_{N} \mid \dot{\psi}_{N}\right\rangle
\end{array}\right)
$$

where $C_{m n}(t)=-i \int_{0}^{t} E_{m n}\left(s^{\prime}\right)\left[1+c\left(s^{\prime}\right)\right] d s^{\prime}$ and $E_{m n}\left(s^{\prime}\right)=E_{m}\left(s^{\prime}\right)-E_{n}\left(s^{\prime}\right)=\hbar \omega_{m n} t . \omega_{m n}(t)$ is the transition frequency between energy level $n$ and $m .\left|\psi_{m}(t)\right\rangle$ and $E_{m}(t)$ are the instantaneous eigenvector and eigenvalue defined in Eq. (3). Clearly the off-diagonal matrix elements are responsible for the transition between two passages. Our task is to reduce the off-diagonal terms. In the adiabatic representation, the effects of the control are determined by $C(t)$, which is an integral of $[1+$ $c(t)] E_{m n}(t)$ in the time domain [14]. Then it is not the details of $c(t)$, but the above integral determines the control effects. Suppose the average transition frequency can be written as $\left\langle\omega^{c}(t)\right\rangle$ under the control, i.e., $C(t)=\left\langle\omega^{c}\right\rangle t$. The oscillation factor $e^{i \hbar \omega_{m n} t}$ now becomes $e^{i \hbar\left\langle\omega_{m n}^{c}\right\rangle t}$. $\left\langle\omega_{m n}^{c}\right\rangle>\omega_{m n}$ if $\langle c(t)\rangle>0$. The propagator can be approximately written as $U(\xi)=e^{-i \int_{0}^{\xi} H(t) d t}$ for infinitely short time $\xi \rightarrow 0$. The evolution now depends on the integral of the product $\left\langle\psi_{m} \mid \dot{\psi}_{n}\right\rangle$ and $e^{-i C_{n m}(t)}$. Generally $\left\langle\psi_{m} \mid \dot{\psi}_{n}\right\rangle$ varies slowly compared with $e^{-i C_{n m}(t)}$, 
according to the Riemann-Lebesgue lemma, the integral of the product of a fast varying and slowly varying function approximately equals to zero. Big $C(t)$ causes the integral to be zero, and all the off-diagonal terms vanish, the adiabatic evolution is obtained.

\section{CONCLUSIONS}

We have introduced an effective control scheme to accelerate the adiabaticity in a non-adiabatic regime by applying a sequence of external pulses upon the Hamiltonian. We use a 1D XY spin chain as an example. By studying the dynamic process of cutting the long chain into two short chains, we find that the magnon will only be in one of the two chains for adiabatic evolution in the single-excitation subspace. The adiabaticity can be induced by applying a sequence of pulses, whether it is regular or random. This can be explained by writing the time-dependent Hamiltonian $H(t)$ in the adiabatic basis. As a result it is the integral of the control function $c(t)$ in the time domain, not the control function itself determines the effectiveness of the control. This results greatly relax the choice of pulses and make it easier to realize in an actual experiment.

\section{Acknowledgments}

This material is based upon work supported by National Science Foundation of China (Grant Nos. 11475160, 61575180), the Natural Science Foundation of Shandong Province (Nos. ZR2014AM023,ZR2014AQ026).
[1] M. Born and V. Fock, Z. Phys. 51 (1928)165.

[2] A. Messiah, Quantum Mechanics (North-Holland, Amsterdam, 1962).

[3] E. Farhi, J. Goldstone, S. Gutmann, J. Lapan, A. Lundgren, and D. Preda, Science 292 (2001) 472.

[4] P. Zanardi and M. Raseti, Phys. Lett. A 264 (1999) 94.

[5] A. Mizel, arXiv:quant-ph/14037694.

[6] K. Eckert, O.Romero- Isart, and A. Sanpera, New. J. Phys. 9 (2007) 155.

[7] L.-A. Wu,Y.-x. Liu, and F. Nori, Phys. Rev. A 80 (2009) 042315.

[8] V. Balachandran and J. Gong, Phys. Rev. A 77 (2008) 012303.

[9] V. Srinivasa, J. Levy, and C. S. Hellberg, Phys. Rev. B 76 (2007) 094411.

[10] B. Chen, W. Fan, Y, Xu, Y.-D. Peng, and H.-Y. Zhang, Phys. Rev. A 88 (2013) 022323.

[11] S. Longhi, EPL, 107 (2014) 50003.

[12] U. Farooq, A. Bayat, S. Mancini, and S. Bose, Phys. Rev. B $91(2015) 134303$.

[13] H. Wang and L. -A. Wu, Sci. Rep. 6 (2016) 22307.

[14] P. V. Pyshkin, D.-W. Luo, J. Jing, J. Q. You, and L.-A. Wu, arXiv: quant-ph/150700815.

[15] T. Kadowaki and H. Nishimori, Phys. Rev. E 58 (1998) 5355.

[16] J. Brooke, D. Bitko, T. F. Rosenbaum1, G. Aeppli, Science 284 (1999) 5415.

[17] B. Heim, T.F. Rnnow, S.V. Isakov, M. Troyer, Science 348 (2015) 6231.

[18] M. V. Berry, J. Phys. A: Math. Theor. 42 (2009) 365303.

[19] X. Chen, I. Lizuain, A. Ruschhaupt, D. G.-Odelin, and J.G. Muga, Phys. Rev. Lett. 105 (2010) 123003.

[20] M. Demirplak and S. A. Rice, J. Phys. Chem. A 107 (2003) 9937.

[21] J.S. Melinger, S.R. Gandhi, A. Hariharan, J.X. Tull, W.S. Warren, Phys. Rev. Lett. 68, 2000 (1992).

[22] J.S. Melinger, S.R. Gandhi, A. Hariharan, D. Goswami, W.S. Warren, J. Chem. Phys. 101, 6439 (1994).

[23] V.S. Malinovsky and J.L. Krause, Eur. Phys. J. D 14 (2001) 147.
[24] F. T. Hioe, Phys. Lett. A 99 (1983) 150.

[25] P. Marte, P. Zoller, and J. L. Hall, Phys. Rev. A 44 (1991) 4118.

[26] H. Theuer, R. Unanyan, C. Habscheid, K. Klein, and K. Bergmann, Opt. Express 4 (1999) 77.

[27] U. Gaubatz, P. Rudecki, M. Becker, S. Schiemann, M. Klz, and K. Bergmann, Chem. Phys. Lett. 149 (1988) 463.

[28] M. Weitz, B. C. Young, and S. Chu, Phys. Rev. Lett. 73 (1994) 2563

[29] A. D. Boozer, A. Boca, R. Miller, T. E. Northup, and H. J. Kimble, Phys. Rev. Lett. 98 (2007) 193601.

[30] Kumar, K. S. et al., Nat. Commun. 7 (2016) 10628.

[31] Ignacio R. Solé, Jesús Santamaría, and Vladimir S Malinovsky, Phys. Rev. A 61 (2000) 043413.

[32] E. Heesel, B. M. Garraway and J. P. Marangos, J. Chem. Phys. 124 (2006) 024320.

[33] B. M. Garraway, K.-A. Suominen, Phys. Rev. Lett. 80 (1998) 5

[34] J. Jing, L.-A. Wu, T. Yu, J. Q. You, Z.-M. Wang, and L. Garcia, Phys. Rev. A 89 (2014) 032110.

[35] Attached file in arXiv: quant-ph/1412.1722 by L.-A. Wu.

[36] M. Lewenstein et al., Advances in Physics 56 (2007) 243.

[37] S. Pasini and G.S. Uhrig, J. Phys. A 43 (2010) 132001.

[38] M. J. Biercuk, H. Uys, A. P. VanDevender, N. Shiga, W. M. Itano, and J. J. Bollinger, Nature 458 (2009) 23.

[39] V. Boyer et al., Phys. Rev. A 73 (2006) 031402(R).

[40] C. Becker, Nat. Phys. 4 (2008) 496.

[41] Z.-M. Wang, L.-A. Wu, M. Modugno, W. Yao, B. Shao, Sci. Rep. 3 (2013) 3128.

[42] Z.-M. Wang, L.-A. Wu, M. Modugno, M. S. Byrd, T. Yu, and J. Q. You, Phys. Rev. A 89 (2014) 042326.

[43] P.W. Anderson, Phys. Rev. 112 (1958) 1900.

[44] Z.-M. Wang, M.S. Byrd, B. Shao, J. Zou, Phys. Lett. A 373 (2009) 636.

[45] Z.-M. Wang, C. A. Bishop, M. S. Byrd, B. Shao, and J. Zou, Phys. Rev. A 80 (2009) 022330.

[46] L.-A.Wu, M. S. Byrd, and D. A. Lidar, Phys. Rev. Lett. 89 (2002) 127901. 